\title{
LE MONDE DRAMATIQUE DE BALZAC
}

\author{
Raul José Machado
}

Le drame se prépare comme un orage qui menace ce monde théâtral, sur cette scène où l'histoire est un drame et les personnages des acteurs. Style d'épopée, comme dans l'Iliade - («c'est la colère, déesse, que tu vas chanter!» - ton de héraut, annonciateur du schéma (pas encore dynamique) de l'oeuvre, par lequel Balzac cherche à attirer notre attention sur le noyau central, en donnant d'avance les lignes de l'action, celles qui permettent au lecteur de prèvoir la force de son lyrisme constructif, de même qu'un plan d'architecture contient déjà en soi la grandeur de l'édificice futur.

Aimant la prose qui s'étale mais qui, avant de se répandre, annonce sa grandeur prochaine, il détestera «la prose rompue» de Stendhal; une prose «qui se prive de l'art d'annoncer" (1) "qui continue dans la voie de la petite phrase de Voltaire».

Ce style annonciateur déclanche l'attente chez le lecteur, entraîné dans un mouvement qui va croissant. Balzac règle l'accélération de ce mouvement par le secret des évènements et le mystère des personnages qui, vus de l'extélfeur, sont de véritables trous d'ombre.

Balzac a le goût du mystère. Les critiques, qui se vantent de voir clair dans un roman balzacien affirment que

1) Article de presse de Balzae a propos de «La Chartreuse» chez Robinson Alain, Lecteur de st, et Bal, 
Balzac ne cache rien, qu'il dit et explique tout. Pourtant Proust, un des plus sensibles et intelligents lecteurs de Balzac, affirme: «aussi est-on étonné de voir que cependant il y a de beaux effets de silence dans son oeuvre!... et moi, je m'étonne bien plus des dessous de l'oeuvre de Balzac: vous connaissez Rastignac? Vrai?!... (2).

Oeuvre remplie par les mystéres du coeur et les secrets d'affaires, elle annonce déjà le secret proustien chez Lucien de Rubempré et Vautrin, personnages dans lesquels Proust a bien vu "les charmes cachés qui affleurent à la surface de IA vie par d'imperceptibles ondulations" (3).

C'est qu'au milieu de ces «imperceptibles ondulations», Balzac quelquefois s'égare et tâtonne dans l'obscurité de son univers, comme si sa création lui échappait et commençait à vivre, d'une existence indépendante et aussi mystérieuse que l'univers réel, après le septième jour de la création d'un Dieu. devenu impuissant pour la comprendre.

Dans Honorine, par exemple, rien n'est expliqué: «ce drame est sans parole et cette obscurité est ici le sujet même. On le sent, ce mystère, comme on sent une caverne sous aes pieds. .. c'est au lecteur à rétablir ce qui remplit le silenee". (4). Dans "Le Curé de Village", le roman est bâti sur le mystère du coeur, sur le secret qui n'est réellement connu que par deux personnages, et l'énigme reste énigme jusqu'à la fin.

Dans «Le Cousin Pons» il y a, d'une part, tout un côte mystórieux, qui «explique» certains évènements et $y$ est représenté par les sciences occultes, image de l'obscurité essentielle de la nature humaine. D'autre part, il y a le secret des complota, e'ost-à-dire le combat entre les associations d'hommes ot de femmes, les plus astucieux et par conséquent les phua forts, combat dont le sens parait etre l'image du poid

1) Crouat *Centre Bainte- Beaves N, H, E

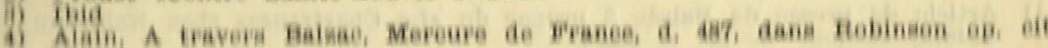

de la société sur cette «cellule social» qu'est l'homme. Le complot y est le portrait collectif, mais réduit, du mystère de l'univers tout entier.

Les hommes s'associent pour la lutte, dans un combat presque toujours inégal, sous le visage impassible des choses, représentées dans ce roman par la collection de tableaux, riche et attirante $d u$ bon musicion.

Dans «Le Faiseur», il y a ce personnage mystérieux, ce Godot qui n'entre jamais en scène, et avec qui pourtant Mercadet tisse toute une toile d'araignée où il veut attraper ses créanciers.

Du mystère jaillit le secret qui va faire rebondir les scènes et créer des péripéties dont la fonction est d'étonner le spectateur devant l'hạbilité du Faiseur qui de lá, c'est-àdire de rien, veut tirer quelque chose.

Dans «La Cousine Bette», le mystère naît de l'hypocrisie sociale des personnages qui, devant certains êtres et dans certains milieux, portent des masques afin de dissimuler tout un autre côté de leur existence, qui ne se révèlera jamais. Dans ce roman, Balzac nous montre l'homme avant tout mêlé aux autres et confondu avec eux. Chaque personnage est ici, plus que dans d'autres oeuvres, concu par rapport à tous les autres; plongé au milieu de cette masse profondément physique et mouvante, il s'y trouve pris, qu'il le veuille ou non, étant soumis au poids de cette structure sociale.

La plupart des personnages balzaciens sont une construction de cette masse sociale, étant conditionnés par la «coquille», dont la «sécrétion» forme leur substance, leur métier, détermine leurs échanges et produits. Le personnage balzacien devient, par conséquent, l'être le moins doté de liberté; il est le jouet des puissances de ce monde rprésentées par l'Argent et le désir de Totalité qu'incarnent certains êtres privilógiós, espèce de meneurs de jeu, d'instigateurs de drame. Chefs de combat, responsables ils entrent "dans cette masse d'hommes comme un boulet de canon, ou s'y glissent 
comme une peste" (5) - tel un Vautrin, dans le "Père Goriot» ou un Frésier, dans «Le Cousin Pons».

Produit de la masse physique et sociale, dès sa présentation, son «portrait», le personnage n'est plus libre, alors que le héros Stendhalien l'est toujours; il est présenté comme une masse solide.

Dans l'univers de Balzac qui se fait dieu il n'y a pas de Ubre-arbitre pour les héros; ils ne peuvent même pas choisir les mots qu'ils voudraient: les mots qu'on trouve sur leurs levres sont ceux de leurs métiers - «chacun pense selon ce gu'll fait». Pas de snobisme de langage comme chez Proust. (anuf pour ceux qui ont fait profession d'être des snobs); le personnage se révèle tout entier dans un seul mot, dans une acule phrase.

Le drame naît de la pression qu'exercent sur le personnage la societé, la politique, le monde extérieur.

Le personnage privilégié, ce personnage soleil éclatant t solide, doué d'une volonté de puissance qui lui permet de braver la société, de lutter contre cette «réunion de dupes ot de fripons», n'est même pas un juge supérieur de l'ordre social dans lequel il vit, mais un produit de cette société, comme le policier.

Tous les héros de Balzac font partie intégrante de cette nociété: le révolté lui-même est un produit, une sécrétion de 1a société, laquelle à la fin triomphe toujours et contre qui tout combat est inutile. Si Balzac admire Rastignac, Vautrin et Fraisier, c'est à cause de leur force, de leur désir de domination et du combat acharné qu'il voit exister en puisanee chez ces êtres privilégiés.

Le drame nait alors de l'impossibilité pour tout homme, peur tout personnage, de sortir vainqueur d'une lutte inégale

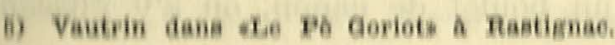

contre ce monstre, ce Paris aveugle à la souffrance, indifférent au malheur du «microcosme humain».

L'action toute seule peut sauver le personnage, ou du moins elle lui donne une consistance, le fait exister: action pour ou contre la société, peu importe. L'important est de ne pas faillir, e'est-à dire de ne pas renoncer à la lutte, au drame, à l'imprévu de l'existence, au mystère.

Si l'élément dramatique des romans et de la piéce en question est fixé par les deux coordonnées, du mystére et de la lutte, l'élément non dramatique de ces oeuvres réside dans l'absence de force du personnage qui, renonçant au combat, tombe dans le statique.

Si Balzac ne met pas ses personnages sur une véritable scène de théatre, il envisage une situation dramatique et théatrale, comme en témoignent tant de passages du Cousin Pons où se trouvent des images empruntées au théatre, des comparaisons avec des situations ou des personnages du théatre classique, ou encore des allussions à des auteurs dramatiques. Le titre qu'il donne au chapitre XXVIII ou Cousin Pons en est un exemple: «L'Or est une chimère (Paroles de Scribe, Musique de Meyerbeer, Décors de Remonencq».

Le roman est plein de comparaisons relatives à des situations théatrales. Les habitants de Paris y sont comparés à une troupe - «le million d'acteurs qui composent la grande troupe de Paris" ( $p .1$ ) ; plus loin, pour attirer l'attention à Paris il faut "offrir une de ces énormités à crever les yeux, comme on dit, et que les acteurs recherchent pour assurer le succès de leurs entrées» (p. 2).

La laideur de Pons devient originale «d'après la grande lei promulguée par Moliere dans le fameux couplet d'Eliante» (p. 11), Et, en décrivant l'affaire Brunner, Balzac regrette: Ah! al cette histoire aval pu se jouer devant le trou du 
souffleur...". A la vue du prétendant, «quelques Parisiens se demandaient d'où sortait la figure profondément tragique de cet Allemand... seul dans une avant-scène c'eût été bien plus beau...» (p. 62).

C'est dans une situation théâtrale que se recontrent les femmes à l'occasion de l'entrevue avec Brunner: «Assez habiles pour éviter les difficultés d'une entrée en scène, les femmes vinrent les premières» (p. 82).

Les expressions «personnage», «joeur un rôle», «scène», *avant-scène», les décors et les dialogues projettent sur une acène idéale les personnages du roman.

En parlant du Ministre de la Justice, du Prèsident, du Procureur Général, à l'occasion de la présentation de Brunner. Balzac les décrit: «Les trois grands personnages acceptèrent, qunique invités à bref délai; chacun d'eux comprit le rôle que leur faisait jouer le père de famille» (p. 88).

L'absence de lucidité chez Pons l'empêche de reconnaitre «les grimaces de la comédie sociale» et le rend incapable d'y jouer un rôle actif (p. 78); en revanche, l'un des rraits qui marquent l'intelligence de Vautrin est justement aon habileté à jouer un personnage différent et à s'accorder, A travers le masque, à la comédie sociale, où il est toujours le personnage parfait et le plus mystérieux.

C'est par un des procédés les plus communs chez Balunc que les personnages de ce roman sont comparés à d'autres personnages qui ont une existence théatrale complète, g'est-Â-dire enfermée dans le contexte d'une piéce de théatre A action, du grand théatre classique de Molière ou de Thakespeare: e'est ainsi que Schmucke, voyant son sublime Pons maltraité par ses familiers: *(il) éprouvait I'indignation d'Aleeste» (p. 100). C'est par ce même procédé que Remoneneq, «devenu comedien... est Harpagon..., (i) 108), Plus loin: zon ne se figure pas les ruses à la Heapin, les toura à la Sganarelle et les séductions à la Florine qu'inventent les chineura: (p, 111),
La Cibot est peinte comme une affreuse «Lady Macbeth de la rue...» (p. 170). Et en voyant l'horreur sur la figure de Schmucke, elle se "leva fière comme Tartufe" ( $p$. 264). D'ailleurs Madame Cibot «joue un si grand rôle dans le drame...» dès le «moment de son entrée dans cette scène» (p. 19).

C. E. Magny constate que l'univers de Martin du Gard est "plus théatral peut-être que romanesque" (6) et elle ajoute: «Non qu'il y ait une nécessaire incompatibilité entre les deux genres: on sait que Balzac, qui n'avait pas renoncé à l'ambition qui le faisait écrire à vingt ans une tragédie sur Cromwell, parle à propos de ses livres des «ouvrages improprement appelés romans» et prèférait leur donner le nom de scènes, qu'ils ont gardé, comme est resté celui de Comédie à l'ensemble de l'oeuvre... L'esthétique de la scène n'en reste pas moins étrange, applioucée à un ouvrage fait pour être lu et non représenté».

Quel lecteur, en effet, n'aurait une vision dramatique du Cousin Pons, devant des scènes où les personnages sont comme projetés hors du roman? Leur dialogue, qu'on entend plutôt qu'on ne le lit, est interrompu ça et là par les paroles de Balzac qui, tantôt nous peint l'attitude de l'acteur ( LLa Cibot fit un geste» ou «Elle regarda Fraisier d'un air égaré» p. 185), tantôt explique ce qui se passe à l'intérieur de son personnage «La Cibot fut effrayée de la perspicacité de cet homme et comprit la raison de la profonde attention avec laquelle il l'avait écoutée» p. 186).

De nombreux dialogues du Cousin Pons sont dramatiques, dans la mesure où le mot désigne un changemet d'état qui marque l'évolution des personnages dans un moment crucial, privilégié et social.

Aux pages 183 sqq. de l'édition Garnier, nous avons l'exemple précis d'un dialogue théatral, qu'interrompent plu-

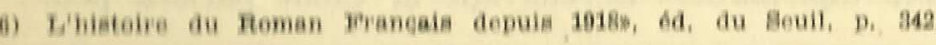


sieurs fois des explications de l'auteur, exigées par le souci de clarté logique auquel Balzac n'hésite pas à sacrifier l'intérêt purement dramatique de ce dialogue qui s'étend sur quatre chapitres (XLV, XLVI, XLVII, XLVIII). Mme. Cibot, conseillée par le Docteur Poulain, va prendre conseil de l'avocat Fraisier, afin de «se faire coucher sur le testament» du bon musicien.

A quoi tient l'intérêt dramatique de ce dialogue? D'abord il faut $y$ noter une intéressante évolution théatraie: un changement s'est opéré à l'intérieur des personnages et par les répliques qu'ils ont échangées, ils se sont révélés à eux-mêmes et au lecteur; d'autre part, à la fin du dialogue, les personnages (Mme. Cibot et Fraisier) se trouvent dans une situation différent de celle où ils se trouvaient l'un vis-à-vis de l'autre au commencement de la scène.

Au début, (prenons la page 185) Mme. Cibot joue la comédie de l'honnête femme scandalisée, que la moindre accusation bouleverse et blesse - «Moi! s'écria la Cibot, moi qui n'ai pas n'une centime à autri!... Moi! Moi!», tandis que Fraisier l'observe froidement! Son regard verdâtre est fort et magnétique, mais «il riait au dedans». Il laisse tranquillement la Cibot parler pendant cinq minutes, il veut l'épuiser et se contente de l'écouter silencieusement. Puis il prend la parole. Ses phrases révèlent son intelligence de stratège. Elles sont courtes et incisives. Par ses propos, il commence à jouer avec les nerfis de sa victime; il la menace. gentiment: «Voulez-vous savoir comment vous pourriez être guillotinée?". A ces mots, la Cibot tombe "pâle comme une morte". On se les représente sur la scène. Fraisier lui montre par des détails comment elle pourrait être menée A la mort. La Cibot, offensée, est agressive. La comédie de thonncte femme scandalisée est finie. Fraisier varie le ton: II devient à la foi aimable, sadique, et grossièrement familier: Allons, ma petite mère...». La Cibot s'effraye et veut quitter la place. Fraisier, qui désormais tient se victime, peut à aon gró changer de position: il parle franchement mala avee diplomatie, Dorénavant la Cibot parlera peu.
C'est un dialogue dramatique parce qu'il marque l'évolution de deux personnages également sordices mais différemment intelligents. Le dialogue suggère les gestes et attitudes des personnages. Les mots se détachent du texte avec les inflexions de voix et le ton des répliques. Les indications nécessaires à un metteur en scène sont aussi dans le texte: la Cibot, d'abord débout, en entendant le mot «guillotinée» tombe théâtralement, et sa physionomie change. Fraisier toujours debout s'incline quelquefois sur sa victime: il est impérieux, il grandit.

Le Cousin Pons reste fortement ordonné autour des évènements, c'est-à-dire autour des moments sociaux que Balzac nous donne presque toujours sous la forme du style direct. C'est dans des moments privilégiés, un bal, une «réunion pour affaires», que l'action avance dramatiquement, impliquant une durée abstraite sans rapport de nature avec le temps romanesque du récit. Cependant, dans l'ensemble du roman balzacien, le cours du temps, en tant que tel, est considérablement plus sensible que dans le théatre. Mais quelques scènes dialoguées chez Balzac sont tellement attachées aux procédés de la technique théatrale, qu'elles en tiennent aussi la durée: cette durée qui si condense aux yeux du spectateur en une série de «noeuds d'évènements».

Dans Le Cousin Pons, l'affaire Brunner contient une scène ( $p .86-87$ ) dont la durée évoque certains moments de «Our town» de Wilder. Balzac, avec beaucoup d'habilité, $\mathrm{y}$ fait des variations sur un thème. Mme. Camusot reçoit quelques personnes qui l'interrogent sur le mariage de sa fille, sur le prétendant. La présidente se livre à un monologue banal fait de phrases juxtaposées, dont le vocabulaire est de «bonne bourgeoisie». Mais si la présentation de la scène est bien artificielle en égard à la façon dont Balzac procède habituellement, le personnage y est classique: Mme Camusot est comme un type de Moliere, elle représente la «more de familie qui marie richement sa fille». Les autres personsages - Balzac pouvait se passer de les nommer -$\|=0$ 
n'interviennent que pour exciter l'idée fixe de Mme. Camusot qui vit son rêve, son obsession, sa morale.

Un autre dialogue qui doit beaucoup à la technique dramatique est celui qui réunit les comédiens, c'est-à-dire les hypocrites Fraisier et Mme. Camusot (p. 213-214): «ce que l'on y dit est tout le contraire de ce que l'on pense». La tochnique théatrale prête à Balzac son jeu scénique. On entend le ton des répliques, on évoques les gestes, le lecteur reconnait, enfin, une scène comique. Ce roman tient du théâtro l'emploi des gros effets comiques dont l'objet est de détondre les nerfs du spectateur. Balzac tire de cette espòce de dialogue d'intérêt psychologique et dramatique les effots les plus saisissants; il l'adapte aux circonstances dramatiques, sans le sacrifice de sa présence, et sous une forme légère et souriante, ça et là, il en commente les conséquences.

Fraisier, averti par la Cibot de l'existence de la riche collection Pons, vient chez Mme Camusot obtenir de cette dame la permission nécessaire pour agir en son nom à l'ouverture de la succession, qui ne doit pas tarder.

C'est la comédie que se jouent deux hypocrites différents: Fralster est hypocrite pour ne pas effaroucher l'honneur de Mme Camusot. L'hypocrisie de Mme Camusot est pour alns dire naturelle. Fraisier, plus intelligent qu'elle, est plus fin. Dans son discours où il constate l'échec de toutes les tentatives faites pour sauver Fons, il y a du cynisme (II dit le contraire de ce qu'il veut faire entendre) mêlé d'hypooriale parfaite: \&Il devait faire renvoyer cette femme (Mme Clbot) ... qui pour avoir trente mille francs, le pousserait dans la fosse..., . Par ce procédé de la contradiction Halase nous donne le nógatif du plan conçu par l'habile stratềe qui vient ì la fols sonder Mme la Présidente et lui dómontrer la ruse de son plan d'opérations.

L'habileté de Fraisier démasque un peu l'hypoerite Mme Camaset. „Dans dix semaines la sucession sera ouvertes eat unvéritable amot de théatres, et de même le mouvement de la présidente, qui en est la conséquence: «La présidente baissa les yeux». Les mots se détachent du texte avec les inflexions de voix qui en doublent le sens, en leur conférant une valeur dramatique.

L'histoire de la captation de l'héritage du Cousin Pons est fortement ordonnée autour des dialogues où viennent se cristalliser les moments les plus dramatiques du roman.

C'est toujours, chez Balzac, à travers un dialogue, dans un moment privilégié et en société qu'éclate le drame qui se joue entre les personnages. C'est au milieu d'une scène dialoguée, dans la troisième partie des Illusions Perdues, que Balzac nous fait connaitre les raisons du drame qui oppose le père Sechard et son fils David. Et c'est Petit-Claud qui nous le révèle: «Tenez, papa Sechard, vous êtes jaloux de votre fils. Ecoutez la vérité! vous avez mis $\mathrm{Da}$ vid dans la position où il est, en lui vendant votre imprimerie trois fois ce qu'elle valait et en le ruinant pour vous faire payer ce prix usuraire. Oui, ne branlez par la tête... Vous haïssez votre fils non seulement parce que vous l'avez dépouillé, mais encore parce que vous en avez fait um homme au-dessus de vous. Vous vous donnez le genre d'aimer prodigieusement votre petit-fils pour masquer la banqueroute des sentiments que vous faites à votre fils et à votre bru... Vous aimez ce petit gars là pour avoir l'air d'aimer quelqu'un de votre famille et ne pas être taxé d'insensibilité..." (7)

Le drame entre père et fils ainsi brusquement révélé finit par la rupture définitive, plus loin, lorsque David, seul cherche l'appui de son père qui, aveuglé par l'avarice, le lui refuse. «Le père et le fils se quittèrent brouillés».

Le dialogue établi entre eux suffit à leur donner d'une part, pour chacun d'eux, la révélation de soi-même, et d'autre part la connaissance de l'autre, par l'intermédiaire du mouvement dramatique imprimé à la scène par Balzac.

7) Lea Misaiona Perdues, 
L'un des traits les plus significatifs du «riche rembourrage» qui donne au personnage balzacien l'épaisseur - cette "troisième dimension romanesque» - qui lui est propre, nous est révélé par le langage particulier à certaines figures du roman, comme Mme Cibot et Schmucke.

Le langage de l'astucieuse et agissante protectrice des deux «casse-noisettes» est tout spécial, ce n'est même pas aeulement le langage des portières: «êlle prodiguait les $\mathrm{N}$ dans son langage» (p. 46).

L'auteur de «l'histoire du Roman Français depuis 1918», parlant des dons spécifiques du romancier, dit: «Le romancler-né possède (entre autres dons) une aptitude congénitale A percevoir la bêtise humaine et à là sténographier pour nous, A nous la restituer dans toute son inane ingénuité».

Dans le Cousin Pons, Balzac nous transmet toute li lourdeur d'un Schmucke par le simples procédé de la transcription phonétique de son langage. Et C. E. Magny Ajoute: «Ce. don n'est pas autre chose qu'un mimétisme, apparenté à celui de l'acteur et qui se traduit très souvent par li capacité du romancier, à un certain moment, de rédiger quelquer chose en un style entièrement différent du sien proDrow, Et elle donne pour Balzac, entre autres, l'exemple de la chronique dramatique que l'auteur des «Illusions Perdues» b́crit pour son Lucien de Rubempre: «On entre, ou Bort, , B. Balzac mime le langage parlé ou le style de ses persennages. II disparaît derrière ce «mimétisme apparenté A celui de l'acteur» en même temps qu'il surcharge son roman d'une épaisseur semblable à la vie. Par ce procédé de style molns écrit que parlé, il mime une illusion auditive en réclamant, A travers le rythme saccadé et particulier à chacun de ве日 personnages, la prósence auditive du lecteur.

Les personnages sont ainsi placés sur une scòne idóale: Ii parlent les uns avee les autres, sous l'éclairage oú le metteur en seène Balase les a mils, au milieu d'un décor solg. neusement peint at déjấ découvert aux yeux du leoteur-apeo tateur avant l'engagement dramatique,
Quant au personnage, avant que le dialogue ne se déclenche, avant que vous ne le voyions agir et lutter, il existe déjà, solide et épais. C'est quand il a été méticuleusement présenté que Balzac le laisse parler. Et quand c'est une Mme Cibot ou un Schmucke qui parle, on sent à quel point certains personnages balzaciens sont détachés de leur créateur; l'argot ou le charabia qu'ils parlent leur assurent une indépendance presque absolue vis-à-vis de l'auteur qui les a créés.

Cette objectivation des personnages par la transcription de leur langage parlé, les place dans un milieu semblable à celui où Pirandello placera son personnage dramatique. Il doit vivre d'une vie parfaitement autonome et détachée de son auteur. L'auteur dramatique ne doit donc montrer que l'action, comme le fait précisément Balzac, parce qu'il n'y a pas de différence d'essence entre le théatre et le roman d'action. La spécificité n'est pas dans le thème, elle est dans le traitement formel. 